\title{
A SIMPLE APPARATUS FOR THE DIAGNOSIS AND RECORDING OF DIPLOPIA*
}

BY

\author{
ERIC C. COWAN \\ Belfast, Northern Ireland
}

AN accurate and simple charting of clinical findings is always essential, and for purposes of record and comparison this applies especially to the recording of ocular muscle palsies. The usual way (and very often the only way) in the orthoptic or consulting room is to use some form of the Hess screen test. To do this requires at least five things of the patient:

(1) Normal colour vision to distinguish between red and green.

(2) A reasonable degree of co-operation and level of intelligence (often lacking in the very old and very young).

(3) The ability to wear the red and green close-fitting goggles, which is often difficult, e.g. in cases of post-herpetic neuralgia.

(4) Good lighting if the thread object is used, and a dark room if the red and green streak lights are to be seen.

(5) Fair visual acuity to be able to see the relatively weak colours of either threads or lights.

These requirements all apply equally to the Lancaster screen test.

Having found myself unable to chart the diplopia fields in colour-blind patients, I designed and made a relatively simple instrument for the purpose, which clinical trials have shown to be quite accurate and efficient. No patient has as yet been unable to use it for any of the five reasons given above. It has worked well with the colour-blind, and a child of 3 years old gave a result that was quite consistent with the clinical findings of a superior oblique paresis presenting as a torticollis.

The apparatus is similar in principle to the well-known cheiroscope, and the end is achieved by using a mirror to superimpose the two fields. One eye sees a bright point of light and the other a tangent screen, the light being seen through a mirror set at an angle of $45^{\circ}$ in front of the viewing eye. The light is thus projected on to the screen which the other eye is viewing directly. As there is no stimulus to fusion the relative positions of the eyes can be charted, with one fixing a given standard point and the other taking up a position relative to it but governed only by muscle tone and balance.

The apparatus is small and simple, easy to use, and quite portable. The tangent scale is etched on white plastic board and pen marks can easily be removed from it after use. The target board is set at right angles to this and a mirror is positioned between the two making an angle of $45^{\circ}$ to each, with the reflecting surface towards the target. In the target are set nine holes 
at the nine standard positions of gaze, corresponding to the test points of the Hess screen (Fig. 1a).

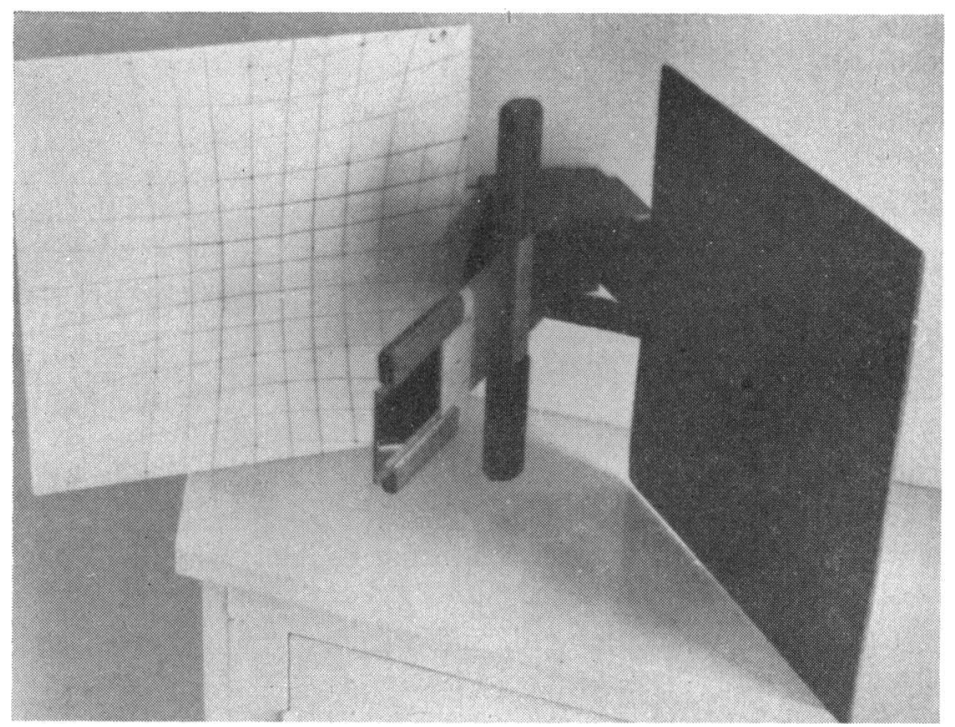

FIG. $\quad 1(a)$. -The tangent screen is to the left, the mirror with the two handles behind it is in the middle, and the target screen is on the right. The nine holes on the screen are not visible.

The patient holds the screen with the tangent chart parallel to his face, looking through the mirror at the points as they are lighted by the examiner, and with his free hand he points to the apparent position of the light on the tangent screen (Fig. 1b).

Fig. $1(b)$. -The patient is holding the apparatus in her right hand and using the left to point to the target point on the screen, which is then marked on the screen by the examiner.

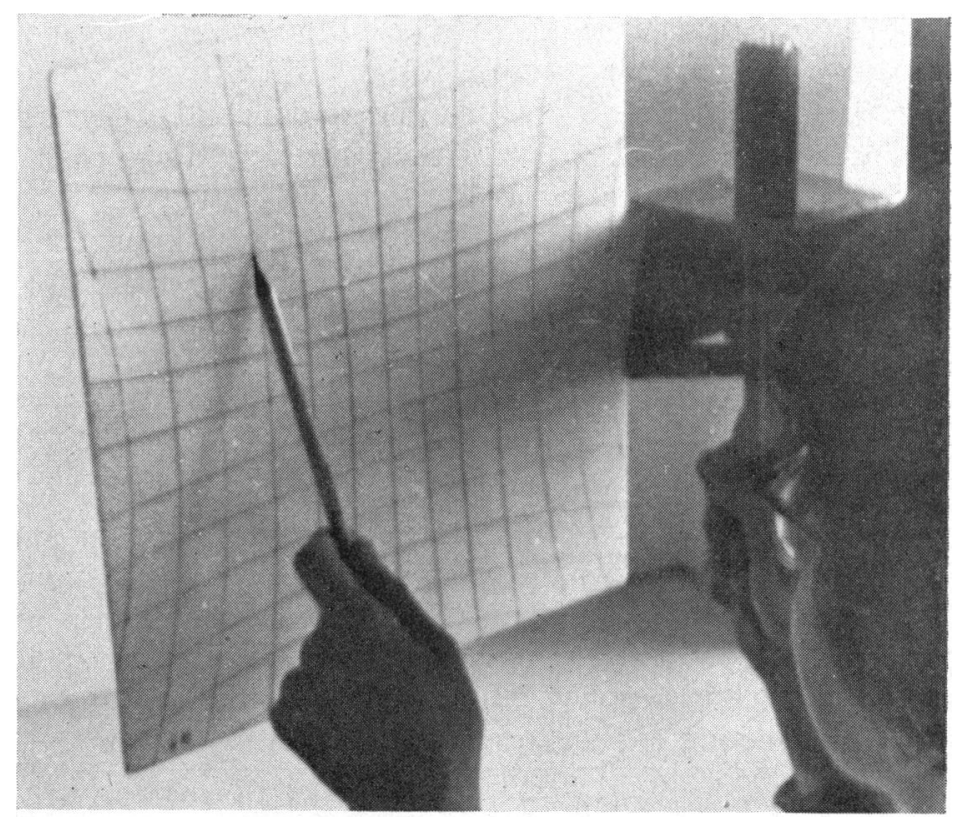




\section{DIAGNOSIS OF DIPLOPIA}

This point is then marked by the examiner with a pen, using red ink when the right eye is under test and blue for the left eye. The examiner lights up the fixation points with a pocket torch, and the nine positions can be quickly covered and recorded. As the chart is absolutely symmetrical, it is quickly inverted for testing the other eye. Nothing is required of the patient except to point to a bright light which appears to him on the tangent screen in front of his face. It is only necessary to tell a child to touch the light, and this produces the required result even in a patient who is only 3 years old.

The nine points being dealt with for the two eyes, the tangent screen is detached and the points marked upon it are easily transferred to a standard Hess chart. If only a diagnosis is required, the smaller of the figures obtained by joining the two sets of points is seen and the muscle (or muscles) affected and the side involved can be read directly (Fig. 2). The marks can then be wiped off the plastic and all is ready for use again.

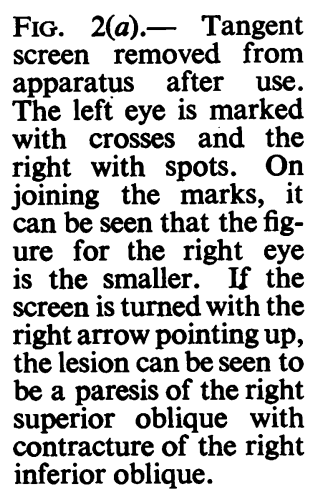

FIG. 2(a).- Tangent screen removed from The left eye is marked with crosses and the right with spots. On joining the marks, it can be seen that the figure for the right eye is the smaller. If the screen is turned with the right arrow pointing up, the lesion can be seen to be a paresis of the right superior oblique with inferior oblique.

FIG. 2(b).-The two figures transferred to recordLEFT ing charts.
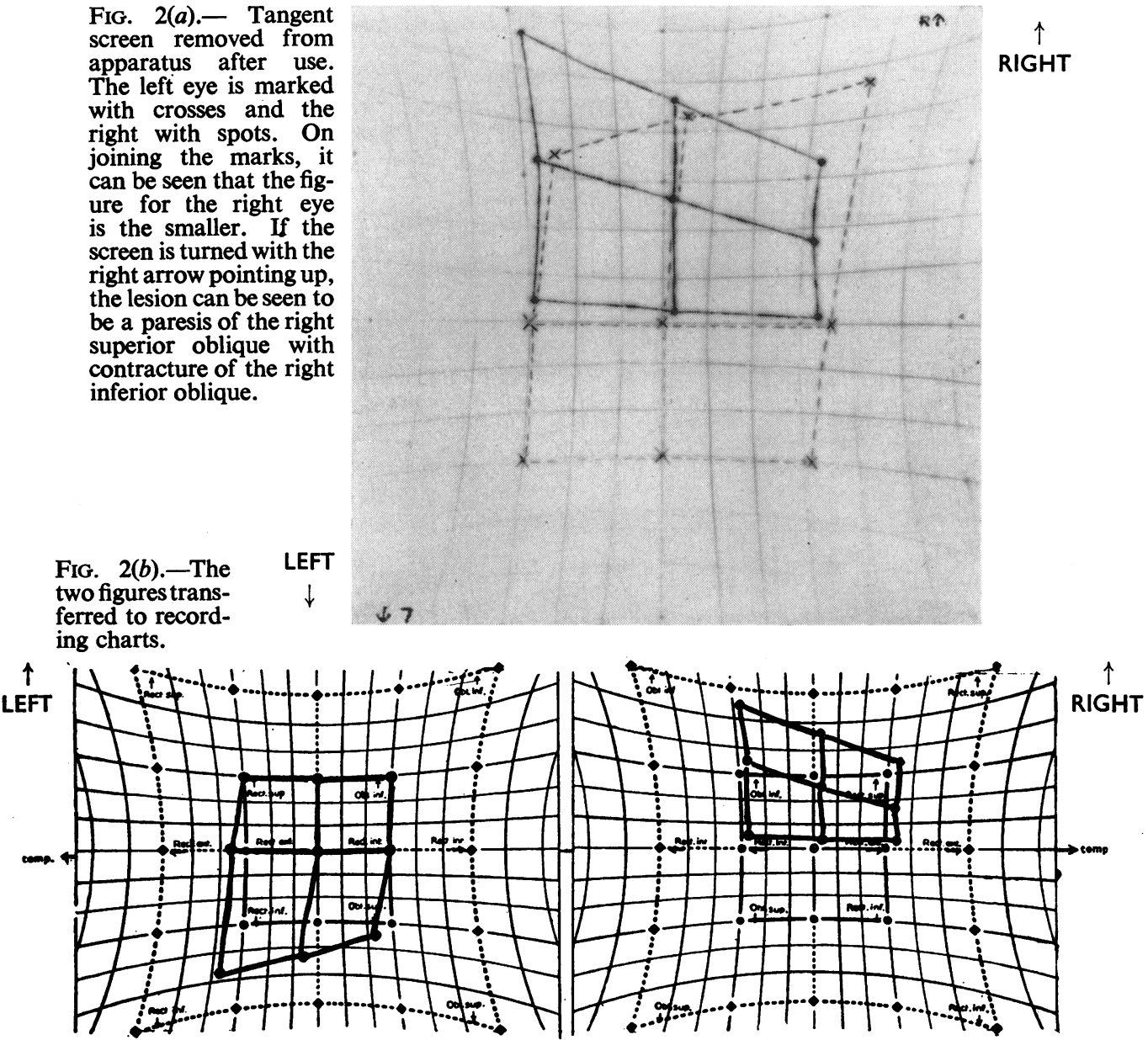
The advantages of such an apparatus are as follows:

(1) It is simple to use and read. The eye being tested is the one looking at the tangent screen.

(2) It requires no appreciation of colour (a faculty not possessed by some 3 per cent. of men).

(3) Little concentration is required on the part of the patient, and this little for at the most just over a minute.

(4) No apparatus other than the unit itself is required.

(5) It can be used anywhere and can easily be taken to the patient if this is more convenient.

(6) The charted results are in effect the same as those obtained by using any of the standard Hess tests, and can be directly compared with Hess charts to check subsequent progress or previous diagnosis (Fig. 3).
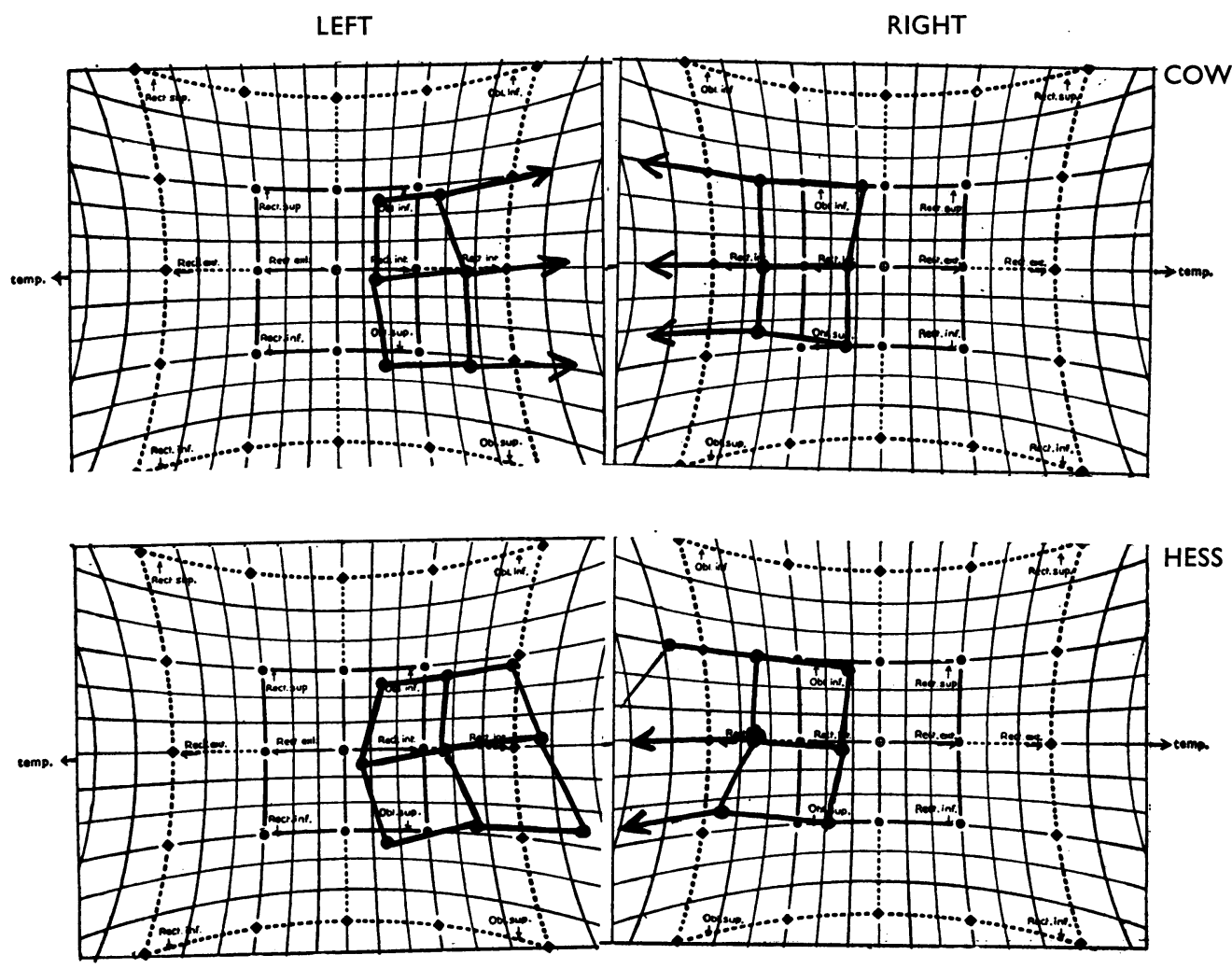

FIG. 3.- The two pairs of charts were done one after the other by different observers and, although not identical, both give the same result. That the two methods are comparable can be clearly seen by this comparison; the upper chart is done by the Cowan method, and the lower by the Hess method. 
(7) The target light can be made very intense to overcome low visual acuity.

(8) No part needs to touch the face, so that pressure on tender or infected regions is eliminated.

Only one small point has to be kept in mind. The two charts as recorded on the screen are one upside down and the other the right way up, and the screen must be correctly orientated when being read (Fig. $2 a$ ).

I have presented the above account of my apparatus which has proved itself of clinical use, in the hope that it might be of interest to those concerned with the diagnosis of muscle and nerve anomalies of the eye in patients for whom the standard diagnostic and recording apparatus cannot be used.

I should like to thank the surgeons of the Benn Hospital, Mr. A. Corkey, Mr. R. H. Baird, and Mr. V. A. F. Martin, for the help given to me in examining their patients and for their constructive criticism of the results obtained. 